\title{
Highly venomous sea kraits must fight to get their prey
}

Received: 21 September 2009/Accepted: 4 January 2010/Published online: 20 January 2010

(C) Springer-Verlag 2010
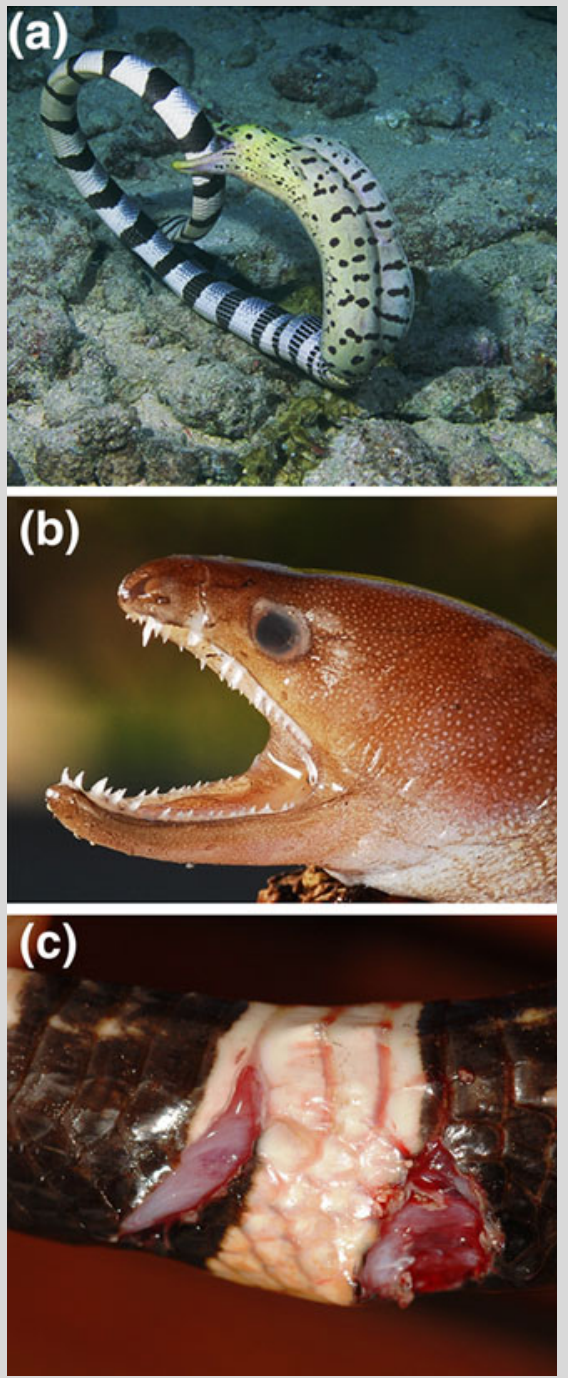

Fig. 1 a An adult female sea krait (Laticauda colubrina) swallowing a large moray eel (Gymnothorax fimbriatus). The eel bit the snake in an (unsuccessful) attempt to escape (R. Lang). This picture also illustrates the fact that sea kraits can swallow their prey tail first. b The head of a freshly regurgitated Gymnothorax eurotus found in the stomach of a specimen of L. saintgironsi; the sharp teeth are clearly visible (X. Bonnet). c A male L. saintgironsi caught at its

arrival on shore, with two rows of deep cuts (X. Bonnet)

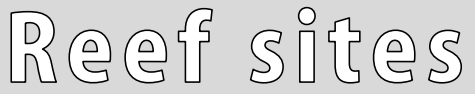

A long-term recapture study in New Caledonia has shown sea kraits (Laticauda laticaudata and $L$. saintgironsi) have a strong predator prey relationship with anguilliform fish (Brischoux et al. 2007; Ineich et al. 2007). Considering krait versus prey body size (sometimes almost of the same size), many conger eels (e.g., Conger cinereus) and moray eels captured by the sea kraits have the potential to retaliate. We observed typical "V-shaped" injuries on many snakes: $29.7 \%$ L. laticaudata had scars and $11.4 \%$ recent injuries $(N=2,797$ individually marked snakes); $33.2 \%$ L. saintgironsi had scars and $9.5 \%$ recent injuries $(N=3,582)$.

Deep cuts sometimes resulted in very large and deep wounds $(N>100)$. Because anguilliform fish are indeed capable of inflicting dangerous bites, the trophic relationships between these two predators are more complex than assumed: the benefit of maximising the amount of energy per foraging trip associated with the capture of large prey trades off against the injury risk that increases with prey size. Prey selection, searching effort, killing tactics, and venom efficiency selection can all be affected.

Figure 1a (20 m depth, island of Taveuni, Fiji, November 2004) clearly illustrates the ability of moray eels to bite sea kraits (see Fig. 1b) during predation as a defence mechanism. We regularly observed sea kraits with recent deep "V-shaped" bites coming on shore without prey in the stomach, possibly to rest and recover (Fig. 1c). Perhaps capturing fish is more difficult than previously assumed, even for the highly venomous sea kraits.

\section{References}

Brischoux F, Bonnet X, Shine R (2007) Foraging ecology of sea kraits (Laticauda spp.) in the Neo-Caledonian lagoon. Mar Ecol Prog Ser 350:145-151

Ineich I, Bonnet X, Brischoux F, Kulbicki M, Séret B, Shine R (2007) Anguilliform fishes and sea kraits: neglected predators in coral-reef ecosystems. Mar Biol 151:793-802

X. Bonnet $(\bowtie) \cdot F$. Brischoux

Centre d'Etudes Biologiques de Chizé, CNRS UPR 1934,

79360 Villiers en Bois, France

e-mail: bonnet@cebc.cnrs.fr

F. Brischoux

School of Biological Sciences A08, The University of Sydney, Sydney,

NSW 2006, Australia

R. Lang

Lafayette, CA, USA

e-mail: rlang76116@aol.com
Coral Reefs (2010) 29:379

DOI $10.1007 / \mathrm{s} 00338-010-0588-3$ 